\title{
Teaching and Learning Exploration of Network Programming Technology Course Based on PBL
}

\begin{abstract}
Wei Wang ${ }^{1, a}$
${ }^{I}$ School of Computer Science and Engineering, Xi'an University of Technology, Xi'an, Shaanxi, China

awellwang@126.com

ABSTRACT

In the teaching and learning of network programming technology, the theoretical knowledge of students is well mastered, but the programming ability is insufficient. Aiming at this problem, a course design scheme based on PBL model is proposed. Firstly, the teaching and learning outcomes are defined. Then, the teaching and learning activities are carried out by combining theoretical teaching with discussion and practical teaching with problems as the starting point. Finally, the actual learning outcomes are assessed by the formative assessment and the summative assessment. In this design scheme, project practice begins with theory teaching simultaneously, the learning hours of practical teaching are increased, and the formative assessment is emphasized. Students can understand knowledge and exercise programming ability in problem solving and project practice.
\end{abstract}

Keywords: PBL model, project practice, teaching and learning design, network programming technology

\section{基于 PBL 的网络编程技术课程教学探索}

\section{王伟 1 , a}

\author{
I 计算机科学与工程学院, 西安理工大学, 西安, 陕西, 中国 \\ awellwang@126.com
}

\section{摘要}

网络编程技术课程教学中, 学生理论知识掌握较好, 但程序设计能力不足。针对这一问题, 提出了一 种基于 PBL 模式的课程教学设计方案。首先定义教学目标; 然后以讨论式的理论教学和以问题为出发 点的实践教学相结合进行教学活动; 最后采用过程性评价和终结性评价对学生实际的学习结果进行评 价。该方案中, 项目实践与理论教学同时进行, 加大了实践教学的学时, 并注重过程性评价, 学生在 问题求解和项目实践中理解知识, 锻炼程序设计能力。

关键词: $P B L$ 模式, 项目实践, 教学设计, 网络编程技术

\section{1. 引言}

网络编程技术是软件工程、网络工程和物联网工程等计算 机类专业的必修课程。该门课程主要学习 Socket 函数和 基于 TCP/UDP 的客户/服务器程序设计。目前, 大多数软 件系统都是在网络环境下运行的,培养学生网络软件开发 能力尤为重要。这门课程需要学生先期掌握操作系统、网 络原理、网络协议分析和程序设计语言等知识, 对学生的 知识基础要求较高, 课程本身的内容也比较多 [1]。近几 年的教学实践表明, 学生的理论知识掌握较好, 但 “不会 编程”。编程类课程的目标是促使学生掌握将问题转换为 解决步骤的方法, 并用程序设计语言编码实现, 这对于计 算机类专业的学生至关重要[2]。培养学生的网络程序设 计能力是本门课程的本质任务。PBL (Problem-Based Learning, 基于问题的学习) 是一种 “以问题为出发点、 以项目来组织” 的教学模式[3]。学生通过分析、解决实
际问题和完成项目来掌握理论知识, 培养学生解决问题、 主动学习和团队合作的能力。这种在项目中学习的教学方 式, 能够有效地培养学生的网络程序设计能力。

\section{2. 教学中存在的主要问题}

\section{1. 学生深入学习的动力不足}

网络编程技术涉及的先修知识较多, 需要学习的 Socket 函数门类多, 数量大。在进行网络编程时, 需要编写客户 和服务器两个程序, 且程序之间要协调一致, 相互通信, 编写和调试程序的难度顿时增加。教师仍然采用讲解和程 序演示的方式进行授课, 没有对不同的函数和不同的客户 /服务器程序结构进行系统分析和比较。教师与学生的交 流和互动较少 (包括课堂上和课堂外)，上课玩手机的学 生较多, 学生的参与度和热情较低。考试形式比较单一, 
队合作能力。网络编程技术课程的教学基于 PBL 模式进 行。

或不愿进行深度学习。

\section{2. 难以培养学生的程序设计能力}

教师注重理论知识的讲解, 只是将函数使用方法和程序结 构 “灌输” 给学生, 没有针对实际问题进行分析和算法设 计, 也没有引导学生一步步编程。在实践环节, 多是要求 学生输入课本上的程序, 查看运行结果或者对课本上的程 序进行少许的改动, 这种验证型或者简单的设计型实验很 难培养学生求解问题和程序设计的能力。久而久之, 学生 对编程产生了 “恐惧感” 和 “焦虑感”。许多 “焦虑” 的 学生选择了去校外培训班练习编程。这里面固然有课程学 时紧张的原因, 但教师在教学中没有注重培养学生的程序 设计能力也是重要的原因之一。

\section{PBL 的主要特征}

PBL 于 20 世纪 60 年代由加拿大麦克马斯特大学医学院 发起, 逐步被世界许多大学和教育机构采用。丹麦奥尔堡 大学所有的教学活动均基于 PBL 模式[4]。PBL 教学模式 主要有三大特征。

(1) 以问题为出发点, 开始学习过程。问题来自于校外 合作机构或现实生活中, 必须是非结构化的, 具有一定的 复杂性, 不能通过简单的方法来解决 [5]。学生在分析问 题和求解问题的过程中完成知识的学习。这种以解决实际 复杂问题为导向的学习方式, 能够将理论和实践紧密结合 起来, 有利于激发学生深度学习的动力。

(2) 以项目来组织, 完成问题的求解。项目小组一般由 6 8 位学生和 1 2 位指导教师构成。学生通过阅读文献、 互相学习和小组讨论等形式合作完成项目, 提交项目报告 和项目过程分析报告。这种学习和求解问题的组织形式有 利于培养学生的团队合作、沟通和表达能力。这些能力在 软件开发中是必须的。

(3) 以学生为中心, 开展学习活动。学习过程中, 学生 是学习的主体, 自主学习, 教师是引导者和帮助者。在课 堂教学中, 教师不是灌输知识, 而是启发学生, 和学生一 起讨论, 一起学习。在项目小组中, 学生自主管理项目, 自主决定项目的组成要素; 教师检查项目工作符合课程的 要求。这种以学生为中心的教学方式, 能够激发学生学习 的主动性、创造性和内在潜力, 培养学生主动学习的能力。 PBL 模式能激发学生自主学习和深度学习, 能够培养学 生分析和解决复杂问题的能力。同时, 也能增强学生的团

\section{4. 教学设计}

网络编程技术的教学要从面向内容向面向能力转变。教学 设计基于 Biggs 的构建一致性理论 (Constructive Alignment) 进行, 主要分为三步: 定义教学目标, 选择 合适的教学方法和评价学习结果 [6]。这三者之间要具有 一致性, 即教学方法要促使教学目标的达成, 评价实际的 教学结果是否达到了教学目标。此外, 要对教学活动和教 学效果进行评估, 持续改进。

\section{1. 教学目标}

首先, 需要定义明确的教学目标。教学目标有两种分类体 系: SOLO ((Structure of Observed Learning Outcome) 和 Bloom[7]。在计算机类专业中, 一般采用 Bloom 分类体 系定义教学目标[5]。Bloom 体系将教学目标分为: 记忆、 理解、应用、分析、评估和创新等六个层级。本课程的教 学目标定义为应用级, 即学生应具有应用知识的能力。具 体的教学目标如下:

LO1: 掌握基本的 socket 函数, 具有设计和开发客户与迭 代服务器程序的能力;

LO2: 掌握 TCP 客户/服务器程序的基本结构, 具有设计 和开发健壮的并发服务器程序的能力;

LO3: 掌握 UDP 客户/服务器程序的基本结构, 具有设计 和开发可靠的 UDP 客户和服务器程序的能力;

LO4：掌握基本的 $\mathrm{I} / \mathrm{O}$ 复用技术，具有设计和开发 TCP/UDP 多协议客户和服务器程序的能力;

LO5: 具有撰写软件开发相关文档的能力, 并能清晰陈述 文档的主要内容。

\section{2. 教学方法}

传统的教学方法是教师讲解课程内容, 学生上机练习编 程。课程的总学时一般是 36 学时左右, 讲课学时和上机 学时的比例大致是 2:1 1:1[8]。理论和实践都不够深入, 学完了课程, 学生不会编程。基于 PBL 模式, 本课程采 用理论教学和项目实践相结合的方式进行。以笔者所在学 校为例, 本课程的教学计划如表 1 所示, 总学时 40 学时, 其中理论教学 16 学时, 实践教学 24 学时。

表 1 网络编程技术课程教学计划

\begin{tabular}{lccl}
\hline \multicolumn{1}{c}{ 教学内容 } & 学时 & 支撑的教学目标 & \multicolumn{1}{c}{ 教学方式 } \\
\hline $\begin{array}{l}\text { 课程简介 } \\
\text { 简单客户和迭代服务器程序基本结构 } \\
\text { TCP/UDP 协议 }\end{array}$ & 3 & LO1 & 讲授、程序演示、课堂讨论 \\
\hline 基本套接口函数 & 2 & LO1 & 讲授、程序演示、课堂讨论 \\
\hline TCP 并发服务器程序基本结构 & 4 & LO2、LO5 & $\begin{array}{l}\text { 讲授、程序演示、小组讨论、小 } \\
\text { 组讲解 }\end{array}$ \\
\hline $\begin{array}{l}\text { I/O 复用技术 } \\
\text { 客户和迭代服务器程序基本结构 }\end{array}$ & 4 & LO1、LO4、LO5 & $\begin{array}{l}\text { 讲授、程序演示、小组讨论、小 } \\
\text { 组讲解 }\end{array}$ \\
\hline
\end{tabular}


以问题为出发点、项目实践、小 组完成

\begin{tabular}{lccl}
\hline 客户和迭代服务器程序设计 & 8 & LO1、LO4、LO5 & $\begin{array}{l}\text { 以问题为出发点、项目实践、小 } \\
\text { 组完成 }\end{array}$ \\
\hline $\begin{array}{l}\text { UDP 客户和服务器程序基本结构 } \\
\text { 名字解析 }\end{array}$ & 2 & LO3 & 讲授、程序演示、小组讨论 \\
\hline 多协议客户和服务器程序基本结构 & 1 & LO4 & 讲授、程序演示 \\
\hline 多协议客户和服务器程序设计 & 16 & LO2、LO3、LO4、LO5 & $\begin{array}{l}\text { 以问题为出发点、项目实践、小 } \\
\text { 组完成 }\end{array}$ \\
\hline
\end{tabular}

\subsection{1. 理论教学}

理论教学侧重于重点和难点知识的学习, 采用课堂讲授、 程序演示和讨论等方式进行, 其中部分课堂讲授采用 “翻 转课堂” 形式。以 Windows 操作系统的 “与 Internet 时间 服务器同步” 为开场白, 引导学生思索 Windows 是如何 从时间服务器上获取时间的, 一步步编写一客户程序访问 时间服务器 time.windows.com, 调试、运行程序, 返回当 前时间。进一步启发学生, “我们能不能自己编写一个时 间服务器呢? ”, 学生进行讨论, 说出自己的实现方法。 教师引导、总结, 编写时间服务器程序。这样, 一个简单 的客户和服务器程序就完成了。通过这一日常工作中的实 例将学生引入网络编程的大门。

理论教学中, 要通过小组讨论和师生讨论, 将功能相同或 相近的协议、函数和程序结构进行比较和归纳。例如, 将 TCP 和 UDP 协议进行比较, 将 inet_aton 和 inet_pton 等 函数进行比较。accept、getpeername 和 recvfrom 等函数 均能获取对端的 IP 地址, 这三个函数分布在课本的不同 部分, 对它们进行比较和归纳。相似地, 还有 close 和 shutdown 函数等。TCP 和 UDP 客户程序的结构及功能有 相同之处, 也有比较明显的区别, 对它们进行比较和归纳, 特别是它们之中 connect 函数的作用要进行详细的比较。 基于 select 和 poll 函数的迭代服务器程序结构比较相近, 均有一 client 数组, 对它们进行分析和比较。理论教学学 时较短, 在学生掌握重点和难点知识的基础上, 要对知识 的逻辑脉络进行总结。从课程的整个知识体系来说, TCP 编程是沿着单向客户和迭代服务器、双向客户和并发服务 器、 $\mathrm{I} / \mathrm{O}$ 复用 (select 和 poll 函数)、基于 $\mathrm{I} / \mathrm{O}$ 复用的双向 客户和迭代服务器进行讲解的; 从单向交互到双向交互, 从迭代服务器到并发服务器, 再到迭代服务器, 此时的迭 代服务器已经得到了升华, 能够处理任意数目的客户连接 (实际上, 连接数目是受系统资源限制的)。理清知识脉 络, 可以使学生既看见树木又看见森林, 灵活运用知识。 信息技术对学生理解抽象知识和激发学生的学习动力有 重要的作用。客户与服务器的交互过程宜用动画的形式进 行展示。本门课程是基于 Linux 平台的, 常用的计算机是 Windows 平台, 可以利用云服务器进行程序演示。近几 年, 笔者即使用两台异地的云服务器进行程序演示, 一台 作为客户端, 一台作为服务器端, 创造真实的网络程序运 行环境。即时互动与反馈系统 (Interactive Response System, IRS) 可以及时掌握学生的听课状态, 增加与学 生的互动。课前, 教师将问题输入系统; 课堂上, 将答题 网址共享给学生, 学生利用手机输入答案, 答案实时显示 在讲台屏幕上; 课后, 教师可以下载答题数据, 并对答题 数据进行分析。教师可以采用 IRS 与学生进行交互, 激 发学生的学习动力, 掌握学生的学习状态。

\subsection{2. 实践教学}

本课程有两个单元的实践环节: 客户和迭代服务器程序设 计及多协议客户和服务器程序设计。这两个 “题目” 只是 实践环节的总体要求, 即问题解决方案中要用到 “题目” 要求的知识。实践环节是以问题为中心的, 题目是开放的, 学生可以在教师提供的问题列表中选择问题, 也可以自己 选取实际工作和生活中的问题进行解决。鼓励学生将大学 生创新创业训练计划项目或 “互联网+” 大学生创新创业 大赛等科技活动中的内容作为选题。在课程开始时即进行 选题, 选题原则上不能重复。

学生以项目小组为单位完成问题分析、算法设计和程序编 写。项目小组由 6 8 位学生和 1 位指导教师组成。建议 以宿舍为单位组成项目小组, 这样便于讨论。学生自主管 理项目进度, 指导教师是引导者和帮助者。实践教学和理 论教学是同时进行的, 学生一边学习基本理论, 一边完成 项目。在项目进行期间, 学生不但要利用课程本身的学时, 还要利用业余时间。指导教师每周与项目小组面对面讨论 一次。由于指导教师人数较少, 讨论室缺乏, 利用即时通 信工具为每个项目小组建立群组，共享资料和在线讨论 [9]。指导教师每周至少与项目小组在线视频讨论一次。 在效果上, 在线讨论和面对面讨论是一致的 [10]。 本课程利用较多时间进行项目实践, 培养学生网络程序设 计能力, 使学生 “会编程”, “喜欢编程”。

\section{3. 学习结果评价}

本课程采用过程性评价和终结性评价对学习结果进行综 合评价, 查看学生是否达到了教学目标, 两者分别占总成 绩的 $70 \%$ 和 $30 \%$ 。

过程性评价由理论教学评价和实践教学评价组成, 两者分 别占总成绩的 $10 \%$ 和 $60 \%$ 。理论教学评价主要评价在理 论教学过程中, 小组讨论结果的汇报情况。实践教学评价 主要评价项目完成情况，由程序质量评价、项目报告评价、 项目汇报评价和小组互评组成，它们分别占总成绩的 $35 \% 、 10 \% 、 10 \%$ 和 $5 \%$ 。程序质量评价主要评价程序功 能、运行状况、程序结构和代码规范程度等。项目报告评 价主要评价报告的逻辑性和规范性，报告中必须有伪代 码。项目汇报评价主要评价学生的表达能力和回答问题情 况。小组互评是指两两小组之间互相审阅对方的代码, 给 出分值。这实际上是一种互相学习的方式。

终结性评价相当于传统意义的期末考试, 本课程采用机考 的形式进行, 考查学生对基本 Socket 函数和基本程序结 构的掌握情况。过程性评价是以小组为单位进行的, 对学 生个体来说, 不一定全面。终结性评价针对学生个体进行, 与过程性评价相结合, 对学生的学习结果进行综合评价。 对学习结果进行评价, 一方面是为了衡量学生的学习效 
the e-tutor in synchronous online problem-based learning: A study in a Master Public Health Programme[J]. British Journal of Educational Technology, 2018, 49(3): 385-397.

\section{4. 教学效果评估}

在教学活动进行中或结束后, 要通过问卷调查、座谈和评 价数据分析等活动, 对教学效果进行评估, 对教学设计进 行反思和修改, 持续提高教学质量。

\section{5. 结论}

网络编程技术是一门实践性很强的课程, 要求学生能够分 析实际问题, 编程解决实际问题。本门课程的教学基于 PBL 模式, 注重发挥学生的主动性, 在讨论中学, 在项 目实践中学, 使学生 “会编程”, “喜欢编程”, 培养学 生的网络程序设计能力。这种教学模式对传统的教师讲授 为主的模式进行了改变, 需要学生积极参与到课堂教学 中, 同时加大了实践教学的学时。在具体教学实践中, 要 灵活运用, 并根据教学反馈, 及时改进、完善。

\section{参考文献}

[1] 胡静, 赵雷, 罗宜元, 等. 网络工程专业的网络编程 课程教学与改革[J]. 计算机教育, 2014(18): 35-38.

[2] Martins VF, de Almeida Souza Concilio I, de Paiva Guimarães M. Problem based learning associated to the development of games for programming teaching[J]. Computer Applications in Engineering Education, 2018, 26(5): 1577-1589.

[3] Askehave I, Linnemann Prehn H, Pedersen J, etc.. PBL-Problem Based Learning[Z]. Aalborg universitet Rektorsekretariatet, 2015.

https://www.aau.dk/digitalAssets/148/148025_pbl-aalborg-m odel_uk.pdf.

[4] Triantafyllou E, Xylakis E, Zotou M, etc.. Applying Learning Analytics in Problem-Based Learning Engineering Semester Projects[C]. Proceedings of the 46th SEFI Annual Conference 2018: Creativity, Innovation and Entrepreneurship for Engineering Education Excellence, 2018: 1328-1335.

[5] Brilingaite A, Bukauskas L, Juškeviciene A. Competency Assessment in Problem-Based Learning Projects of Information Technologies Students[J]. Informatics in Education, 2018, 17(1): 21-44.

[6] Biggs J. Enhancing Teaching Through Constructive Alignment[J]. Higher Education, 1996, 32(3): 347-364.

[7] Meerbaum-Salant O, Armoni M, Ben-Ari M. Learning computer science concepts with scratch[J]. Computer Science Education, 2013, 23(3): 239-264.

[8] 刘琰, 常斌, 陈静, 等. 网络编程技术实践教学方法 的探索与实践 [J]. 计算机工程与科学, 2016, 38(Suppl(1)): 7-11.

[9] Zeng F, Deng G, Wang Z, etc.. WeChat: a new clinical teaching tool for problem-based learning $[\mathrm{J}]$. International journal of medical education, 2016(7): 119-121.

[10] de Jong N, Verstegen D M, Könings K D. The role of 